\title{
Avoidable factors associated with pregnant and postpartum patients admitted to two intensive care units in South Africa
}

\author{
N C Ngene, ${ }^{1}$ Dip Obst, Dip HIV Man, MMed (Fam Med), FCOG, MMed (O\&G); J Moodley, ${ }^{2}$ MB ChB, FRCOG, FCOG, MD; \\ R P von Rahden, ${ }^{3}$ BSc (Lab Med), MB BCh, Adv Dipl Appl Physiol, FCA, Cert Crit Care; F Paruk, ${ }^{4}$ MB ChB, FCOG, Cert Crit \\ Care, MD; P N Makinga, ${ }^{5}$ MB ChB, Dip Obst, Dip HIV Man, Adv Cert Health Man, MPH, MMed (Fam Med)

\begin{abstract}
${ }^{1}$ Department of Obstetrics and Gynaecology, Edendale Hospital, Pietermaritzburg, and School of Clinical Medicine, College of Health Sciences, Nelson R Mandela School of Medicine, University of KwaZulu-Natal, Durban, South Africa

${ }^{2}$ Women's Health and HIV Research Group, University of KwaZulu-Natal, Durban, South Africa

${ }^{3}$ Department of Anaesthesia, Critical Care and Pain Management, Grey's Hospital, Pietermaritzburg, and School of Clinical Medicine, College of Health Sciences, Nelson R Mandela School of Medicine, University of KwaZulu-Natal, Durban, South Africa

${ }^{4}$ Department of Critical Care, School of Medicine, Faculty of Health Sciences, University of Pretoria, South Africa

${ }^{5}$ Department of Obstetrics and Gynaecology, Northdale Hospital, Pietermaritzburg, and School of Clinical Medicine, College of Health Sciences, Nelson R Mandela School of Medicine, University of KwaZulu-Natal, Durban, South Africa
\end{abstract}

Corresponding author: N C Ngene (ngenenc@gmail.com)

Background. Identification and prevention of any avoidable factor (AVF) associated with pregnancy may reduce critical illnesses and the need for intensive care unit (ICU) admission.

Objectives. To determine AVFs that occurred prior to the admission of pregnant and postpartum patients to two ICUs in South Africa (SA) and the resulting maternal outcomes.

Methods. The hospital records of all pregnant and postpartum patients in two public hospital ICUs in Pietermaritzburg, SA, between 1 July 2010 and 30 April 2011 were assessed to identify pre-ICU AVFs. Each patient was followed up until the 7th day after ICU discharge or until hospital discharge (whichever came first), to observe maternal outcomes: survival, death or hypoxic ischaemic brain injury (HIBI).

Results. Of 84 patients assessed, $41(48.8 \%)$ had $\geq 1$ AVF. Patient-related, administrative and health-worker-related AVFs were identified in $32.1 \%(27 / 84), 19.0 \%(16 / 84)$ and $7.1 \%(6 / 84)$ of patients, respectively. The most common patient-related AVF was the commencement of antenatal care after 20 weeks' gestation. Unavailability of ICU beds was the most common administrative AVF. Iatrogenic pulmonary oedema associated with intravenous fluid resuscitation was the most frequent health-worker-related AVF. Of women who had AVFs, 9 (22.0\%) died, 2 (4.9\%) had HIBI and 30 (73.2\%) suurvived. The relative risk of death or HIBI among patients with AVF/s was $1.2(p=0.7)$.

Conclusions. The principal interventions that may prevent AVFs are ongoing community health promotion, strengthening of obstetric skills training on fluid resuscitation and expansion of critical care services.

S Afr J Obstet Gynaecol 2016;22(1):8-12. DOI:10.7196/SAJOG.2016.v22i1.1033

In the 2011 - 2013 triennium, an institutional maternal mortality ratio of 154.06 per 100000 live births was reported in South Africa (SA) ${ }^{[1]}$ Of the 4452 maternal deaths reported, 26.7\% were assessed as being probably avoidable, with an additional $32.8 \%$ possibly avoidable. ${ }^{[1]}$ A large number of these deaths occurred in an intensive care unit (ICU). ${ }^{[1]}$ It has also been reported that $48 \%$ of maternity admissions to a tertiary hospital ICU in New Zealand were potentially avoidable. ${ }^{[2]}$ In the New Zealand study, failure of health workers to recognise the severity of illnesses was the most common avoidable factor (AVF). The situation in SA has not been investigated fully. In an attempt to address the issue of AVFs associated with maternal morbidity and mortality, models for assessing preventability have been reported. ${ }^{[3,4]}$

In SA, the AVFs associated with pregnant and postpartum patients admitted to ICUs are rarely reported separately, despite the contribution of these factors to both maternal mortality and morbidity. Given that ICUs are scarce facilities in $\mathrm{SA},{ }^{[5]}$ the identification of these AVFs may lead to the development of recommendations to address these issues, with a possible reduction in the number of ICU admissions. The number of ICU beds in SA reflects the scarcity of critical care facilities. In 2004 - 2005, the ratio of beds in ICUs and high-care units in the public sector per total SA population ranged from <1:20 000 to 1:80 000. ${ }^{[6]}$ However, in 2010, a report on the availability of ICU beds in different countries suggests that the number of ICU beds per 100000 population was 8.9 in SA, but 24.6 in Germany. ${ }^{[7]}$ The figure was 3.9 ICU beds per 100000 population in China (an upper-middle-income country like SA). ${ }^{[7]}$

Despite the obvious scarcity of ICU resources, there is a paucity of literature focusing strictly on AVFs associated with pregnant women admitted to ICUs, especially on the African continent. The aim of this study was to determine the pre-ICU AVFs, and the maternal outcomes of pregnant and postpartum women admitted to the state hospital ICUs in Pietermaritzburg, SA.

\section{Methods}

A 10-month prospective chart review of all pregnant and postpartum (up to 42 days after delivery) patients admitted to two ICUs (in a regional and a tertiary hospital) in Pietermaritzburg, SA, was undertaken. The two public hospital ICUs are referral centres for the majority of patients (urban and rural) living in the Pietermaritzburg area (uMgungundlovu District Municipality). The population of uMgungundlovu in the years 2013 - 2014 was 
approximately $1052730 .^{[8]}$ In addition, the two hospitals also take referrals from further afield than the uMgungundlovu district, because of limited availability of health services. During the study period, the critical care beds in the tertiary and regional hospitals were 5 and 6, respectively, in the mixed medical-surgical ICUs. There were also an additional 4 beds at the coronary care unit in the tertiary hospital. Data were collected from 1 July 2010 to 30 April 2011.

The study received institutional approval including that of the Biomedical Research Ethics Committee of University of KwaZuluNatal (reference BE 192/09) prior to its inception. The ethics committee waived the need for written informed consent given the severity of illness of the patients and the fact that there were no interventions. A multidisciplinary team of clinicians experienced in the management of critically ill obstetric patients assessed each patient prior to or during the ICU admission. The principal investigator was notified by the medical staff when a pregnant or postpartum patient was admitted to the ICUs. The principal investigator then visited the ICUs at least once a day to collate patients' information and then had discussions with co-investigators to identify AVFs. Collection of patient information was feasible because it was easy to commute between the two hospitals. The principal investigator was a trainee in the Pietermaritzburg hospitals complex but not the most responsible physician managing the patients. The method used for the identification of the AVF is similar to that approved by the National Committee for Confidential Enquiries into Maternal Deaths in SA. ${ }^{[4]}$ In this system, the medical staff involved in the management of the patient also participate in the discussion to identify AVFs. Although there is a risk that the clinicians who managed the patients may be biased, the SA system provides a learning opportunity to prevent a repetition of identified AVFs. Patients were followed up by the principal investigator from the time of ICU admission until the 7th day after ICU discharge or until hospital discharge (whichever came first). Maternal outcomes (survival, hypoxic ischaemic brain injury (HIBI) or death) were observed daily during this follow-up period. The duration of followup was determined by the investigators' previous experiences that suggest limited success in protracted patients' follow-up after hospital discharge at the study sites. It was therefore not possible to determine 28-day all-cause mortality (death that occurred in the first 28 days following a patient's admission to the ICU) ${ }^{\left[{ }^{[9]}\right.}$ In addition, the management of the patients during the study period was not different from the routine clinical practice in the ICUs.

AVFs were defined as preventable events that could directly or indirectly lead to adverse outcome/s in patients' care. Listing all such events (actions and inactions) here will be an attempt to prejudge the future standard of care by disregarding possible innovations that may be introduced in the care of a pregnant woman. The AVFs were categorised as administrative, patient related and health worker related. This categorisation was based on the model approved and utilised for reporting maternal deaths in SA..$^{[4,10]}$ The model does not prescribe comparison of maternal outcomes of patients in different units of a hospital. We therefore did not compare the outcome of this study with those of patients who were not admitted to the ICUs. With use of this model, AVFs that result from a pregnant woman herself, her family or environment are regarded as patient related. ${ }^{[10]}$ Health-worker-related AVFs are actions or inactions of a healthcare professional that could compromise a patient's care. Administrative AVFs are administrative shortcomings that may lead to adverse outcome/s in a patient's care. ${ }^{[10]}$ Patients with any combination of patient-related, administrative or health-worker-related AVFs were regarded as having multiple factors. Furthermore, iatrogenic injuries were assessed as being an AVF if the injury was deemed preventable had the attending medical personnel rendered an acceptable standard of care.

For the purposes of this study, women who commenced antenatal care after 20 weeks' gestation were considered to have booked late and therefore regarded as having an AVF. Booking for antenatal care before 20 weeks' gestation affords the attending healthcare worker the opportunity to detect and attend to pregnancy-related issues early. The initial antenatal visit is recommended to be at 10 weeks' gestation. ${ }^{[11]}$ At 20 weeks' gestation, a structural anomaly scan can be performed. Beyond 20 weeks' gestation, a fetus is potentially viable in well-resourced settings. Moreover, in audits of maternal deaths in SA, patients who initiated antenatal care after 20 weeks' gestation are categorised as having delayed in accessing medical help. ${ }^{[10]}$ The time lag before ICU admission (i.e. the time between definitive identification of the need for a patient to be admitted to an ICU and the time of admission of the patient to ICU) was also documented. In a previous study, a time lag of $>4$ hours was considered as delayed admission because of its association with increased mortality. ${ }^{[12]}$ In the present study, 6 hours was arbitrarily considered to represent enough time to arrange and provide an ICU bed in our setting.

\section{Sample size}

The study duration was predetermined prior to the inception of the study, and it was based on convenience. The number of patients included in the study was therefore determined by the study period.

Over the 10-month study period, 82 patients were admitted to the ICUs. Two additional patients who were admitted to ICU before commencement of the study were still in the ICU when the study commenced. These two were included in the study, resulting in 84 participants.

\section{Statistical analysis}

Data are presented as frequencies, percentages, means and relative risk (RR). In the calculation of RR, death and HIBI are reported as a single outcome because they represent adverse outcomes. Analysis of data was done with SPSS version 22 (IBM Corp., USA).

\section{Results}

AVFs were identified in 41 of 84 study participants (48.8\%). Participants with AVFs were aged between 15 and 43 years (mean 24.5 (standard deviation (SD) 7.4) years). Those without AVFs were aged between 15 and 40 years (mean 25.6 (SD 7.0) years). AVFs were found in $14(56.0 \%), 22(46.8 \%)$ and $5(41.7 \%)$ patients aged $<19$ years, $20-34$ years and $>35$ years, respectively. As explained under 'Methods', the AVFs were categorised as patient-related, health-worker-related and administrative. Table 1 shows the distribution of different categories of AVFs among the various age groups.

The mean gestational age of the patients on admission to the ICUs or when the index pregnancy ended was 28.1 (SD 9.1) weeks. Postpartum patients comprised 31/41 (75.6\%) of those with AVFs and $35 / 43(81.4 \%)$ of those without AVFs. Eclampsia/severe preeclampsia was the most common pre-ICU admission principal diagnosis, in 14/41 (34.1\%) and 13/43 (30.2\%) of patients with and without AVFs, respectively. The other common pre-ICU admission 
Table 1. Distribution of AVFs among the different age groups

\begin{tabular}{lllll}
\hline \multirow{2}{*}{ Category of AVFs } & \multicolumn{3}{c}{ Age groups, $\boldsymbol{n}$ (\%) } \\
\cline { 2 - 4 } Patient factors & $\leq \mathbf{1 9}$ years & $\mathbf{2 0}-\mathbf{3 4}$ years & $\mathbf{2 3 5}$ years & Total, $\boldsymbol{n}$ (\%) \\
Administrative factors & $10(40.0)$ & $9(19.1)$ & $1(8.3)$ & $20(23.8)$ \\
Health-worker factors & $0(0.0)$ & $2(4.3)$ & $0(0.0)$ & $2(2.4)$ \\
Multiple factors & $3(12.0)$ & $6(12.8)$ & $2(16.7)$ & $11(13.1)$ \\
No AVF & $1(4.0)$ & $5(10.6)$ & $2(16.7)$ & $8(9.5)$ \\
Total & $11(44.0)$ & $25(53.2)$ & $7(58.3)$ & $43(51.2)$ \\
${ }^{*}$ Multiple factor is any combination of the following: patient-related, administrative and health-worker-related factors. This excludes \\
the presence of more than one AVF of the same category.
\end{tabular}

\section{Table 2. List of AVFs*}

\begin{tabular}{|c|c|}
\hline \multicolumn{2}{|l|}{ Patient-related factors $(32.1 \%, 27 / 84)$} \\
\hline Started antenatal care after 20 weeks' gestation & 11 \\
\hline Refused treatment of primary medical condition & 5 \\
\hline Defaulted antenatal care & 5 \\
\hline No antenatal care & 3 \\
\hline Delay in seeking treatment & 3 \\
\hline Unsupervised home delivery & 2 \\
\hline Unsafe abortion & 2 \\
\hline Ingestion of herbal concoction & 1 \\
\hline \multicolumn{2}{|l|}{ Administrative factors $(7.1 \%, 6 / 84)$} \\
\hline Delayed ( $>6$ hours) admission to ICU due to unavailability of beds & 4 \\
\hline Lack of blood for transfusion & 1 \\
\hline Hospital staff (including ICU) strike & 1 \\
\hline \multicolumn{2}{|l|}{ Health-worker-related factors $(19.0 \%, 16 / 84)$} \\
\hline \multicolumn{2}{|l|}{ Delayed or suboptimal treatment ${ }^{\dagger}$} \\
\hline Iatrogenic pulmonary oedema due to injudicious use of intravenous fluid & 4 \\
\hline Oxygen not administered despite the need & 1 \\
\hline Insufficient fluid therapy in a severe revealed placental abruption & 1 \\
\hline Delayed intubation despite early need & 1 \\
\hline Delayed abdominal delivery for placental abruption with prolonged labour & 1 \\
\hline Patient fell to floor from hospital bed owing to poor nursing care & 1 \\
\hline Delayed referral of complicated case from district to regional hospital & 1 \\
\hline Delayed treatment due to admission to the wrong ward & 1 \\
\hline Four instead of two doses of betamethasone administered to a patient for fetal lung maturity & 1 \\
\hline \multicolumn{2}{|l|}{ Delayed ability/inability to recognise problem } \\
\hline $\begin{array}{l}\text { Cervix not examined to detect cervical tear during uterine evacuation and repair of } \\
\text { intrapartum perineal tear }\end{array}$ & 1 \\
\hline Massive vaginal bleeding through a uterine pack not detected & 1 \\
\hline Septic miscarriage diagnosed as a threatened miscarriage despite obvious clinical features of sepsis & 1 \\
\hline Proteinuria of $3+$ not investigated to diagnose pre-eclampsia & 1 \\
\hline Delayed diagnosis of uterine rupture despite obvious clinical features & 1 \\
\hline $\begin{array}{l}\text { *AVFs identified in } 48.8 \% \text { of patients ( } 41 / 84) \text {. Some patients had more than one AVF. } \\
\text { †Patients with delayed or suboptimal treatment had the correct diagnosis. } \\
\text { ¥The hospital protocol demands that the cervix is examined during the treatment/repair of obstetric perineal tears. }\end{array}$ & \\
\hline
\end{tabular}

principal diagnoses among the patients with and without AVFs were placental abruption, pneumonia, placenta praevia,
The most common indication for ICU admission in both groups was haemorrhage: $11 / 41$ (26.8\%) and 11/43 (25.6\%) in those with and without AVF, respectively. The additional common indications for ICU admission in all patients were sepsis, respiratory failure, repeated fits and pulmonary oedema.

Table 2 lists the AVFs identified in the study participants. Patient-related AVFs were most frequent, and occurred in $32.1 \%$ (27/84).

The maternal outcomes (survival, HIBI and death) are shown in Table 3. Of the 41 patients with any AVF, $9(22.0 \%)$ died, 2 (4.9\%) had HIBI and 30 (73.2\%) survived. In addition, of the 43 patients without any AVF, 9 (20.9\%) died, 1 (2.3\%) had HIBI and 33 (76.7\%) survived. Among 8 patients with multiple AVFs, $50.0 \%$ died. The RR of death or HIBI among patients with any AVF was 1.2 (95\% confidence interval (CI) $0.5-2.4$; $p=0.7)$.

\section{Discussion}

The ages of patients in both groups were similar. However, AVFs were least common in those aged $>35$ years. A possible explanation for this may be that these women are experienced and therefore likely to utilise the health system effectively; for example, they may be more likely than younger patients to adhere to medical advice, ask questions, etc. These speculative explanations of the healthseeking behaviour of pregnant women aged below and above 35 years need to be investigated further.

In this study, $48.8 \%$ of the patients had an AVF. This is similar to the $48 \%$ found in a similar study in New Zealand. ${ }^{[2]}$ The patient-related AVF category was most common in our study (mainly the initiation of antenatal care after 20 weeks' gestation, that occurred in 11 women). In the New Zealand study, the most common AVF was the failure of healthcare workers to recognise the seriousness or complexity of patients' illnesses. ${ }^{[2]}$ Differences in the disease profile, health system and level of education between SA and New Zealand may account for this disparity. Using community caregivers (who undertake a home visit and provide health education) to identify pregnant women and encourage them to book early for antenatal care will be helpful. Such community engagements may be needed to sustain adequate utilisation of healthcare services available in SA. 
Table 3. Maternal outcomes of patients with different categories of AVF

\begin{tabular}{lllll}
\hline & \multicolumn{3}{c}{ Maternal outcomes } & \\
\cline { 2 - 4 } Category of AVF & Survived & HIBI & Died & Total \\
\hline Patient related, $n(\%)$ & $16(80.0)$ & $0(0.0)$ & $4(20.0)$ & $20(100)$ \\
Administrative, $n(\%)$ & $2(100)$ & $0(0.0)$ & $0(0.0)$ & $2(100)$ \\
Health-worker-related, $n(\%)$ & $9(81.8)$ & $1(9.1)$ & $1(9.1)$ & $11(100)$ \\
Multiple factors, $n(\%)$ & $3(37.5)$ & $1(12.5)$ & $4(50.0)$ & $8(100)$ \\
AVF absent, $n(\%)$ & $33(76.7)$ & $1(2.3)$ & $9(21.0)$ & $43(100)$ \\
Total, $N$ & 63 & 3 & 18 & 84
\end{tabular}

Given that the most common patient-related AVF was the commencement of antenatal care after 20 weeks' gestation, enhancement of the health help-seeking behaviour of women of childbearing age is needed in SA. In a similar study conducted in a tertiary hospital in New Zealand, the inability to recognise the severity of a medical condition by either the patient or her family was the most common patient-related AVF. ${ }^{[2]}$ An audit of all women with severe acute maternal morbidity (SAMM) in Pretoria, SA, showed that infrequent or no antenatal care was the most common patient-related AVF associated with SAMM. ${ }^{[13]}$ Nonetheless, the absence of prenatal care is still a health concern, even in a high-income country such as Canada. ${ }^{[14]}$ It is important to note that patient-related AVFs may be influenced by family, community and patients' personal circumstances. ${ }^{[10]}$ For greater insight into these influencing circumstances, further studies are required to investigate patient-related AVFs adequately. Community health promotion, education and empowerment of women are required to eliminate the patient-related AVFs identified in this study (Table 2).

The most common health-worker-related AVF was iatrogenic pulmonary oedema due to injudicious use of intravenous fluids. Therefore, there is a need to train medical staff on fluid management of critically ill maternity patients. It has been previously reported that substandard management was the most prevalent health-workerrelated AVF among women with SAMM in Pretoria. ${ }^{[13]}$ A study in India indicated that an inappropriate treatment plan was the most common health-worker-related AVF among women with near-misses. ${ }^{[15]}$ In New Zealand, healthcare personnel lack of skills and knowledge in problem recognition ${ }^{[2]}$ have been reported as the most frequent preventable health-worker-related AVFs. The health-worker-related AVF found in New Zealand may be attributed to lack of clinical policies and protocols. ${ }^{[2]}$ The disparity between the health-workerrelated AVFs found in $\mathrm{SA}$ and New Zealand is difficult to explain by a single reason. Differences in patient profile and availability of treatment protocols between the two countries may partly account for the differences observed. The pre-ICU admission diagnoses in the four patients who had iatrogenic pulmonary oedema in our study were septic miscarriage, placenta abruption with no obvious risk factor, and two cases of severe pre-eclampsia. Ongoing training of health workers on topics that relate to the identified AVFs (Table 2) could prevent inappropriate medical care.

The administrative AVFs identified were a lack of blood for transfusion, a strike by hospital staff and delayed ICU admission because of unavailability of beds. The most common administrative AVF was the lack of ICU beds, which suggests that public health institutions in SA are overburdened with high patient loads and that there is a need to improve the capacity of our health services. This finding concurs with that of the SAMM study carried out in Pretoria. ${ }^{[13]}$ Sadler et al. ${ }^{[2]}$ in New Zealand have reported that the most common organisational/management AVF was a lack of clinical policies or protocols. The disparity noted between the two countries may be due to differences in health systems, economic strength, population sizes and/ or political will. Contrary to these findings, transportation difficulties were the major administrative AVF in India among women with near-misses. ${ }^{[15]}$ In the index study, lack of transportation was not identified among the administrative AVFs. It is possible that the workers providing ambulance services have a short response time when caring for critically ill obstetric patients. The speculated short response time may not apply to other patient groups. Nonetheless, this hypothesis needs further investigation.

A high percentage of patients with multiple AVFs died in comparison with other categories (Table 3). This suggests that the contribution of different categories of the AVFs to death may be additive or synergistic. The RR of death or HIBI among patients with any AVF was $1.2(p=0.7)$. Despite the nonstatistically significant $p$-value, care should be taken to prevent AVFs. It is also possible that the patients with AVFs utilised more ICU resources than those without any AVF.

\section{Study limitations and strengths}

The present study was not about patients with SAMM who were not admitted to an ICU. However, maternity cases admitted to an ICU may be a reflection of all cases of SAMM. Owing to the limited literature on pre-ICU AVFs, the findings of the present study have occasionally been compared with cases of SAMM, including those not admitted to ICUs. Unfortunately, patients who had SAMM but were not admitted to ICUs were excluded from the study. This is because it was difficult to track each case of SAMM that was not accepted for critical care at the study hospitals. In addition, any AVF or detailed description that was not documented in patients hospital charts would have been missed. However, it is unlikely that adequate information was not documented in the hospital charts. This is because patients are usually thoroughly reviewed (also by the critical care staff) before ICU admission. The expertise of experienced investigators was utilised to identify the AVFs so as to ensure scientific rigour. Furthermore, owing to other factors such as comorbidities and the relatively small number of patients audited, it is difficult to ascertain the extent to which each AVF (e.g. late initiation of antenatal care) contributes to the need for ICU admission and the resulting maternal outcomes. Another example is the difficulty in establishing the contributory effect of steroid in a patient who was given four instead of two doses of $12 \mathrm{mg}$ intramuscular betamethasone for the management of preterm labour (Table 2). The same patient received tocolysis and also had comorbid valvular heart disease. The quality of critical care may also affect outcomes, but substandard treatment is extremely unlikely in the equipped ICUs because the management of patients in these units is closely supervised by a team of experienced and academically certified intensivists.

Despite these limitations, our study is one of the very few that has focused on preICU AVFs associated with pregnant and 
postpartum patients admitted to ICUs. The suggested interventions based on the findings of this study will be specific to the target population and are therefore likely to be effective. Furthermore, debilitating morbidity such as HIBI was included as an outcome.

\section{Conclusions}

Approximately $49 \%$ of the study participants had an AVF. Ongoing community health promotion, education and empowerment of women are probable measures that will prevent the identified patient-related AVFs. To prevent the health-worker-related AVFs, continuing training and retraining of healthcare personnel, particularly on fluid balance management, should be strengthened by emergency obstetric simulation training. Administrative AVFs may be prevented by promoting donation of blood in the SA population to ensure a regular supply of blood/blood products. Lastly, studies such as ours must be brought to the attention of the National Department of Health so that sufficient ICU beds and welltrained health professionals are made available to reduce maternal morbidity and mortality.

\section{References}

1. National Committee on Confidential Enquiries into Maternal Deaths. Saving Mothers 2011-2013: Sixth Report on the Confidential Enquiries into Maternal Deaths in South Africa. Short Report. Pretoria: NDoH, 2015. http://www.kznhealth.gov.za/mcwh/Maternal/Saving-Mothers-2011-2013short-report.pdf (accessed 28 August 2015).
2. Sadler LC, Austin DM, Masson VL, et al. Review of contributory factors in maternity admissions to intensive care at a New Zealand tertiary hospital. Am J Obstet Gynecol 2013;209(6):549.e1-549. e7. DOI:10.1016/j.ajog.2013.07.031

3. Geller S, Cox S, Kilpatrick S. A descriptive model of preventability in maternal morbidity and mortality. J Perinatol 2006;26(2):79-84. DOI:10.1038/sj.jp.7211432

4. Moodley J, Pattinson R, Fawcus S, Schoon M, Moran N, Shweni PM, on behalf of the National Moodley J, Pattinson R, Fawcus S, Schoon M, Moran N, Shweni PM, on behalf of the National
Committee on Confidential Enquiries into Maternal Deaths in South Africa. The confidential Committee on Confidential Enquiries into Maternal Deaths in South Africa. The confidential
enquiry into maternal deaths in South Africa: A case study. BJOG 2014;121(suppl 4):53-60. enquiry into maternal deaths

5. Buga EC, Nethathe GD, Mathivha L. Obstetric critical care services in South Africa. S Afr J Obstet Gynaecol 2015;21(1):4-5. DOI:10.7196/SAJOG.954

6. Bhagwanjee S, Scribante J. National audit of critical care resources in South Africa - unit and bed distribution. S Afr Med J 2007;97(12):1311-1314.

7. Adhikari NKJ, Fowler RA, Bhagwanjee S, Rubenfeld GD. Critical care and the global Adhikari NK, Fowler RA, Bhagwanjee S, Rubenfeld GD. Critical care and the global
burden of critical illness in adults. Lancet 2010;375(9749):1339-1346. DOI:10.1016/S01406736(10)60446-1

8. uMgungundlovu Health District. District health plan 2015/2016. http://www.kznhealth.gov.za/ Strategic/DHP/2015-16/Umgungundlovu.pdf (accessed 13 March 2016).

9. Abizanda Campos R, Altaba Tena S, Belenguer Muncharaz A, et al. Study of post-ICU mortality during 4 years (2006-2009). Analysis of the factors related to death in the ward after discharge from the ICU. Med Intensiva 2011;35(3):150-156. DOI:10.1016/S2173-5727(11)70019-4

10. National Committee on the Confidential Enquiries into Maternal Deaths. Saving Mothers 2008-2010: Fifth Report on the Confidential Enquiries into Maternal Deaths in South Africa. Comprehensive Report. Pretoria: NDoH, 2012. http://www.sanac.org.za/resources/cat_view/7 publications/9-reports (accessed 27 August 2015).

11. National Institue for Health and Clinical Excellence (NICE). Antenatal Care: NICE Clinica Guideline 62. Issued: March 2008, last modified: December 2014. https://www.nice.org.uk/ guidance/cg62/resources/guidance-antenatal-care-pdf (accessed 28 August 2015).

12. Hung SC, Kung CT, Hung CW, et al. Determining delayed admission to the intensive care unit for mechanically ventilated patients in the emergency department. Crit Care 2014;18(4):485. DOI:10.1186/s13054-014-0485-1

13. Pattinson RC, Macdonald AP, Backer F, Kleynhans M. Effect of audit on critically ill pregnant women. Clin Gov 2006;11(4):278-288. DOI:10.1108/14777270610708814

14. Knight E, Morris M, Heaman M. A descriptive study of women presenting to an obstetric triage unit with no prenatal care. J Obstet Gynaecol Can 2014;36(3):216-222.

15. Taly A, Gupta S, Jain N. Maternal intensive care and 'near-miss' mortalities in obstetrics. J Obstet Gynecol Ind 2004;54(5):478-482. http://medind.nic.in/jaq/t04/i5/jaqt04i5p478g.pdf (accessed 28 August 2015). 